\title{
Executive Laterality: Evidence from Hemisometry, Hemisity, and MRI
}

\author{
Bruce E.,", Morton Stein Rafto ${ }^{2}$ \\ ${ }^{1}$ School of Medicine, University of Hawaii, Hawaii, USA \\ ${ }^{2}$ Kaiser Permanente Hawaii, Hawaii, USA
}

Copyright $\bigcirc 2017$ by authors, all rights reserved. Authors agree that this article remains permanently open access under the terms of the Creative Commons Attribution License 4.0 International License

\begin{abstract}
The bilateral human brain includes structures producing a preconscious executive element, the source of the final decision to act. This executive element appears to be located unilaterally in the brain on the larger side of the anterior cingulate cortex. In a given individual, its side of residence is genetically determined in idiosyncratic manner by a phenomenon called hemisity. Using flash hemisometers, a single $0.5 \mathrm{~ms}$ flash was directed to both cerebral hemispheres of the subject simultaneously. This was done either by stimulating nasal retinal surfaces or temporal retinal surfaces of the subject's eyes. Of 91 subjects, 87 (96\%) reported seeing two flashes; the first appearing on one idiosyncratic side, closely followed by another on the same side. Yet, only single flashes were reported when nasal and temporal retinae were simultaneously stimulated. This non-intuitive two flash result would occur if on one side of the brain the flash went directly to a unilateral executive observer to be seen first. Because the flash going to the other, non-executive side of the brain would not be perceived there, it would have to cross the corpus callosum to reach the executive observer as a delayed second flash. Here, the individual brain side of the executive observer determined by this method was highly correlated with each subject's larger anterior cingulate and MRI-calibrated hemisity. These results provide confirmatory evidence for the existence of executive laterality.
\end{abstract}

Keywords Anterior Cingulate Cortex, Asymmetry, Behavior, Corpus Callosum, Hemisphericity, Familial Polarity

Executive Laterality: Evidence from hemisometry and hemisity. It describes three research findings which we believe demonstrate that an executive element of the brain is unilateral. That is, by use of $0.5 \mathrm{~ms}$ hemisometry light flashes, the executive observer is shown to be located on the same side of the brain as larger side of the anterior cingulate cortex, here revealed by MRI. Further, it is shown to be on the same side as the right or left brain-oriented element of hemisity. The research was conducted in compliance with the Code of Ethics of the World Medical. Association and the Committee of Human Studies at the University of Hawaii Institutional. Review Board and posed no significant risks to participants.

Highlights: When a single $0.5 \mathrm{~ms}$ light flash was directed by a hemisometer separately to each side of the brain, two closely spaced flashes were seen. The first flash appeared on a fixed, but idiosyncratic side that was highly correlated with the larger side of the anterior cingulate cortex, and also on the side of the subject's right or left brain-oriented hemisity, both determined by MRI. The second flash resulted from the delay produced by its having to cross the corpus callosum from the side lacking an executive observer to get to a side where one was present.

\section{Introduction}

Although the brain is structurally bilateral, many functional laterality asymmetries have been found to exist, not only within the cerebral hemispheres, $[1,2]$, but also subcortically $[3,4]$.

Theoretically and by definition, within any functional institution, including the brain, there can be only one executive system element that decides the final non-reflexive response to incoming stimuli. If the above is true, then in the bilateral brain of vertebrates, this single executive system "observer" which operates pre-consciously $[5,6]$, would be expected to be located unilaterally.

The limbic anterior cingulate cortex (ACC) has repeatedly been identified as the site of this brain executive system element $[7,8,9,10,11]$. The anterior cingulate cortex (ACC) is a separated bilateral structure lying on either inner side of the longitudinal fissure of the brain. The two ACC inner surfaces on either side of the midline 
fissure show remarkable individual variations in terms of gyri and sulci $[12,13]$. Furthermore, there have been many reports of functional asymmetry of the ACC $[14,15]$.

Since the 1960s, relief from certain grand mal seizures has been obtained by severing the corpus callosum to prevent the excitation in one cerebral hemisphere from crossing to the other. Investigations of such "split brain" patients have provided evidence for behavioral and even consciousness differences produced by the separated hemispheres [16]. This was proposed to be the source of right vs. left brain syndrome called "hemisphericity" by Bogen [16]. Although the concept of hemisphericity was accepted intuitively by the general public, after hundreds of false starts, unworkable definitions, faulty theories, inaccurate assays, and unproven claims, academic research on hemisphericity collapsed [17]. A major cause for the downfall of hemisphericity was its too proper psychologically quantitative definition placing an individual on a unique point on a gradient between extreme left and extreme right postulated behaviors, where almost everybody ended somewhere in the middle due to natural avoidance of extremes.

Recently, the binary concept of hemisity has been introduced, where one was either born right or left brain-oriented. Such a definition has surmounted the earlier un-testability and other difficulties that led to the demise of hemisphericity. This redefinition states that the behavioral lateralities of hemisity result from the unavoidable existence a single unilateral executive element ("there can only be one bottom line"), which is inherently embedded either on the right or left side of the asymmetric cerebral hemispheres in the region of the anterior cingulate cortex [13]. Apparently, the local environments of the left hemisphere's top-down, important-details processing skills are sufficiently incompatible that they have been separated from the right hemisphere's bottom-up, big picture processing skills by the midline fissure. This confers upon the executive embedded either on the right or left hemisphere with certain behavioral and thinking style differences. These have been detected in five areas: logical orientation, type of consciousness, fear level and sensitivity, social-professional orientation, and pair bonding-spousal dominance style [18].

Two neuroanatomical differences between individuals of left and right hemisity subtypes have been discovered. The first was that right brainers had up to a three-fold larger corpus callosum than left brainers [19]. The second revealed that the larger side of the ventral gyrus of the anterior cingulate cortex was always on the same side as the individual's hemisity [13]. These discoveries support the validity of the hemisity construct and the methods upon which it was developed [20, 21, 22, 13, 14]. It is important to note that this executive element is considered not to be a homunculus, but rather a preconscious survival-optimizing subsystem of the brain.
Here, further evidence for the existence of a unilateral executive "observer" element is reported.

The method used, called hemisometry, was based upon an unpatented invention (Morton, unpublished) derived from studies by Klemm and Efron [25, 26, 27] who demonstrated that the CNS does not correct for differences in nerve path length when estimating event simultaneity. For example, when Klemm, [25] mildly shocked forehead and toe simultaneously, all his subjects asserted that the shock to their foreheads came first.

In the present report, Flash Hemisometers were constructed that simultaneously delivered a $0.5 \mathrm{~ms}$ low intensity flash of light to either both temporal, or both nasal retinas of both eyes.

If there was a unilateral executive observer element, using these hemisometers, it would be predicted to receive a first $0.5 \mathrm{~ms}$ flash from the specific retinal surface that directly shunts the signal to the brain side of its location. Then, a second, delayed $0.5 \mathrm{~ms}$ flash would be expected to arrive at the same observer, having been shunted across the corpus callosum from the other observerless side of the brain, secondarily reaching the side of the unilateral executive at least $30 \mathrm{~ms}$ later [28, 29, 30].

Although there was only one stimulus flash emitted within the apparatus, the subjects of the present study consistently reported observing two sequential light flashes. Importantly, the first flash arrived on an individually characteristic right or left side. The observation was that the resulting apparently random distribution of observer laterality among these subjects was found to be highly correlated with the side of their MRI calibrated hemisity subtype and the larger side of the anterior cingulate cortex [13]. These hemisity, hemisometer, and MRI outcomes strongly support the existence of a unilateral executive element.

\section{Methods and Materials}

\subsection{Subjects}

The subjects of this study were colleagues, graduate students, and others in the University of Hawaii at Manoa (UHM) community. Of the 91 total, 53 were male and 38 female. Among the males, 20 were identified as right brain-oriented males (RMs), as described below, and 33 were left brain-oriented males (LMs). Of the females, 19 were right brain-oriented (RFs) and 19 were left brain-oriented (LFs). Thus, there were 39 right brain-oriented persons (RPs) and 52 left brain-oriented persons (LPs). Their average age was $44.5 \mathrm{yrs}+/-13.7$ S.D. Seventy nine percent of them were Caucasian, and about $10 \%$ were left-handed. The study met the guidelines of the Committee on Human Studies of the University of Hawaii Institutional Review Board and posed no significant risk to participants. 


\subsection{Subject Hemisity Calibrations}

The right or left brain hemisity subtype of these subjects had previously been determined to be essentially congruent with the larger side of their bilateral $\operatorname{vgACC}$ by MRI as follows [13]:

\subsubsection{MRI Setup for Assessment of the Anterior Cingulate Cortex Asymmetries}

MRI assessments were obtained employing a General Electric Signa 1.5 Tesla MRI instrument. A midsagittal plane setup calibration protocol was run for 3 minutes using a $\mathrm{T} 1$ weighted spin echo sequence $(\mathrm{TR}=400 \mathrm{msec}$, $\mathrm{TE}=1 / \mathrm{Fr}$ ) to image $5 \mathrm{~mm}$ thick slices from the midline plane and two adjoining sagittal planes $6 \mathrm{~mm}$ on either side. The in-plane resolution was 860 x 980 microns. Whole-head photographic images (magnification $=0.72 \mathrm{x}$ ) were directly prepared from these three planes. These three exposures were printed on a single $35 \times 43 \mathrm{~cm}$ film sheet for each subject. This procedure enabled both cortical walls of on either side of the midline fissure to be visualized and measured, thus allowing subelement lateralities of the ACC to be evaluated. Digital analysis processing equipment was not available. However, triplicate quantitative measurements were made manually directly from the film with an intra-rater reliability of 0.96 .

\subsubsection{Assessment of the Laterality of the Ventral Gyrus in Areas 24 and $24^{\circ}$ of the ACC}

At two ACC sites on each side of the brain, one in Area 24 and the other at Area 24 [7], estimations of the relative thickness of the ventral gyri $(\mathrm{vgACC})$ there were made. This abbreviation and these four ACC locations within Areas 24 and 24' are not to be confused with the more frontal ventral region of the perigenual ACC. The vgACC locations where these relative thickness estimations were made are illustrated by the arrows in Figure 1. Two lines were extended outward perpendicularly from the inner edge of the $\mathrm{CC}$, ending in one case at a more frontal point in Area 24 and in the other at a more dorsal point in Area 24 . Both points were in the plane of the cingulate sulcus and arbitrarily selected, based upon the sites in the region giving the largest thickness for each brain side involved. There were no points on the film where detectable boundaries were not present (Figure 1). These vgACC measurements extended dorsally to the paracingulate gyrus. This method and the results of the measurements for these subjects have already been published [13].

For about $20 \%$ of the subjects, the upper dorsal edge of the $\mathrm{CC}$, the lower boundary of the vgACC was obscure, as illustrated in the right hemisphere panel of Figure 1B. In order not to exclude these subjects, the always-sharp ventral edge of the $\mathrm{CC}$ was used as the lower reference point and accurate $\mathrm{CC}$ thicknesses were obtained from the other two sharp sections of the three vertical sections $(-6,0$, $+6 \mathrm{~mm})$. This was justified because when the CC boundaries in all three sections were sharp, the thickness along its length was invariant within each subject. These $\mathrm{CC}$ thickness measurements were subtracted from the overall measurement to give two estimates of the relative thickness of the $\operatorname{vgACC}$ on either side of the brain. All measurements were made in triplicate to the nearest $\mathrm{mm}$ by a single estimator. The average of these two lateral relative thickness estimates from the vgACC of each side were then used to determine upon which side of each subject's brain the $\operatorname{vgACC}$ was thicker. L/R ratios were computed. For LPs the ratio was greater that one. It was less than one for RPs [13].

\subsection{Hemisity Determination Methods}

The following six independent hemisity methods, described in detail elsewhere, were used to determine each subject's individual hemisity (mean number of methods/subject was 5.3). The Best Hand Test alone has also been used to investigate the mean hemisity of academic and professional populations ( $\mathrm{n}=1048$; [39].
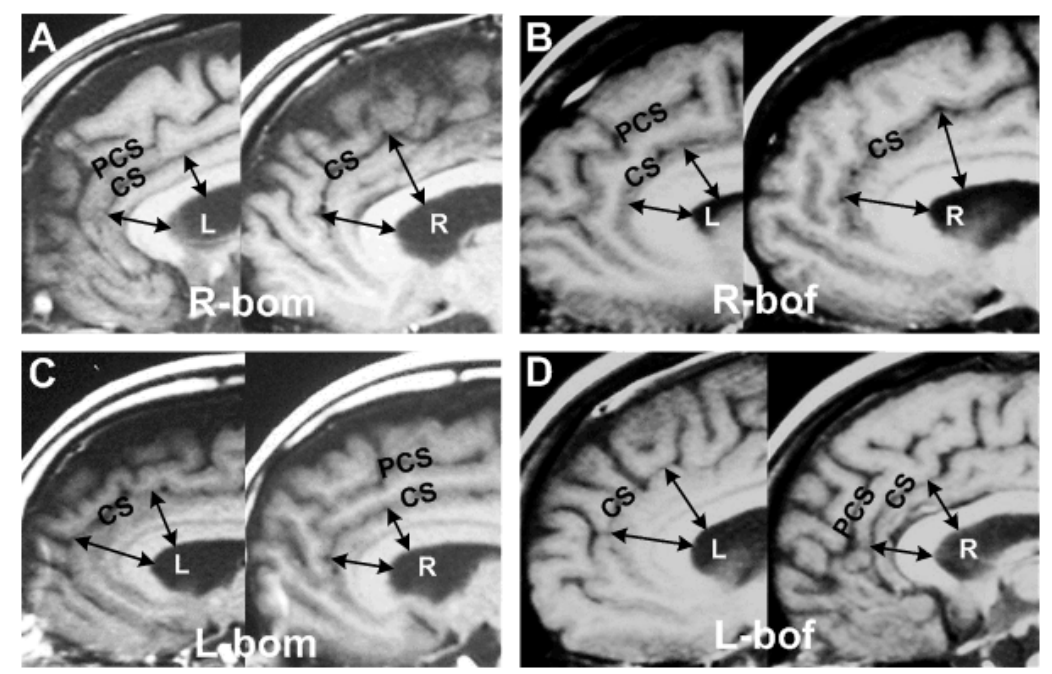

Figure 1. Asymmetries of the Anterior Cingulate Cortex 


\subsubsection{The Dichotic Deafness Test $[22,23,19]$}

Utilized the "Tonal and Speech Materials for Auditory Perceptual Assessment", Disc 1.0 (1992), purchased from the Long Beach Research Foundation. This was used to measure minor ear deafness of 115 pseudo-randomly selected subjects during simultaneous and $90 \mathrm{~ms}$-separated presentations of dichotic consonant-vowel syllables. Attention bias was reduced by instructing subjects to write syllables heard in each ear.

\subsubsection{In the Phased Mirror Tracing [22],}

Mirror tracings of pentagonal stars were produced by both hands of 131 subjects with the aid of a Lafayette Instrument, Mirror-drawing apparatus, Model 31010. Competitive mean elapsed time outcomes between hands were phase-adjusted by use of the Affective Laterality Test [22].

\subsubsection{In the Best Hand Task (Morton 2003b, 2003d)}

Forms containing 20 horizontal lines for each hand to bisect were completed by 142 subjects, measured, phased, and scored according to Morton [23].

\subsubsection{In Zenhausern's Preference Questionnaire [21, 30]}

All subjects ranked 21 statements from "strongly agree

Temporal Retina Flash Hemisometer

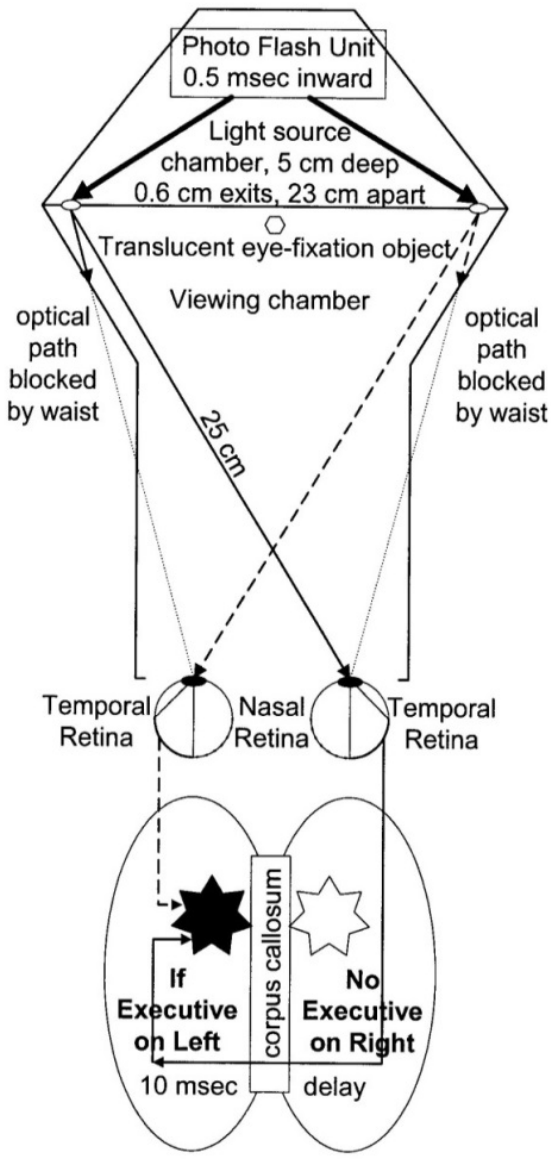

to strongly disagree".

\subsubsection{The Polarity Questionnaire [21] is a binary} questionnaire of 11 statements, which were assessed by all subjects, using true or false answers

2.3.6. In the Asymmetry Questionnaire [25]: all subjects were selected between 15 binary statement pairs

\subsection{Hemisometry}

\subsubsection{Hemisometer Construction}

Prototypes of nasal and temporal retinal Flash Hemisometers were constructed to the inner dimensions illustrated in Figure 2 (top) from about $4 \mathrm{~mm}$ thick plywood by woodshop techniques, using Household "Goop" (Eclectic Products, Inc., USA) for assembly. The outer dimensions of these highly portable (Figure 3 ) units were $35 \times 25 \times 6 \mathrm{~cm}$. An inexpensive Vivitar 16M photoflash unit was firmly attached to either prototype by a strong rubber band looped over protruding screws on each side of the Hemisometer boxes. When the subject pressed the firing button on the flash unit, a flash of $0.5 \mathrm{msec}(1 / 2000$ sec) duration was produced.

\section{Nasal Retina Flash Hemisometer}

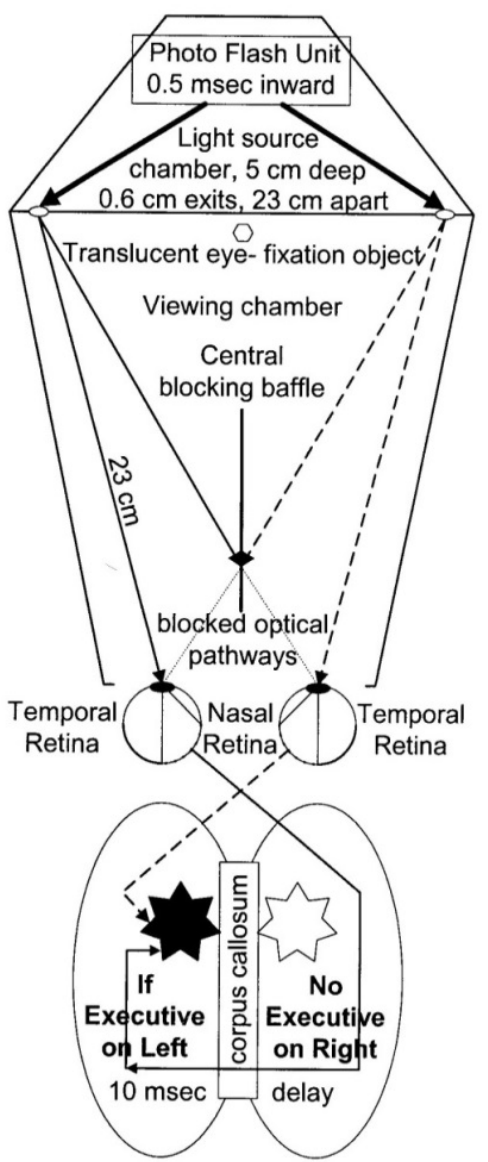

Figure 2. Flash Hemisometer Design and Function, as Illustrated by a Subject with their Executive on the Left 


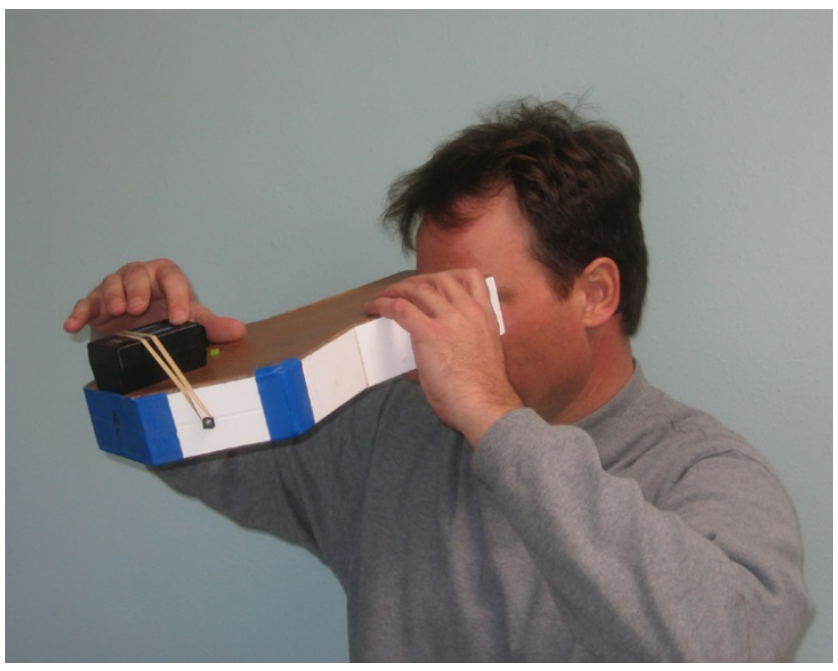

Figure 3. Photo of Flash Hemisometer in Use

\subsubsection{Hemisometer Procedure}

Each subject gave their consent to participate after being informed that they would see a series of low intensity flashes emerging from small right and left holes within the Hemisometer. They were asked to fix their eyes on the central luminous target, and told that their task was to determine from which side the first of two almost simultaneous lateral flashes came and which if any of the flashes was brightest. They were informed that an afterimage of the last occurring flash might, or might not, quench the former or otherwise linger, giving an illusion of prominence [40].

After giving themselves several flashes, ad lib, the subjects using either the nasal or retinal flash hemisometer became able repeatedly to assert on which side the flash appeared first. This side was recorded. Their brain executive observer was postulated to be located on the opposite side, as indicated by the neural path schematic for both hemisometers (Figure 2, $\mathrm{n}=30$ for subjects tested with each). Suggestibility contributions to subject responses were excluded because no flash separations were reported by the subjects $(\mathrm{n}=22)$ when using two placebo hemisometers that either flashed both retinal sides simultaneously or neither side (producing background illumination). Most subjects could directly discern which side came first. Others could not tell which side was first, but felt that the flash from a particular side lingered, or extinguished the former to become brighter overall. A very few indicated that the flash dropped out on one side, leaving only the flash on the other. All these asymmetric responses were lumped together. About 5\% insisted they could not distinguish any difference between sides.

\subsection{Statistical Analysis}

The Statistica 6.0 package was used for scatter plotting and Pearson's correlational analysis.

\section{Results}

In Figure 2, bottom, the schematic path of visual input into the brain is diagramed. There, it may be seen that visual stimuli to either nasal retinae are shunted to the opposite side of the brain for each eye. In contrast, flashes transmitted to the either of the temporal retinae are transferred to the same sides of the brain [16]. Clearly, a corresponding figure could be drawn with the executive positioned on the right.

In Figure 2 top, the schematic design of two flash hemisometers is shown. One utilized only temporal retinal visual inputs from each eye, while the other used only those from the nasal retinae. In either hemisometer, flashes from one side were directed to the contralateral side of the brain, as indicated by the schematic. Figure 3 illustrates a temporal retina flash hemisometer in use.

Usually a difference could be detected by the subject between the sides of the simultaneous flash coming from the hemisometer and be given a temporal order. When subjects were tested with either hemisometer, the side of the flash judged to emerge from the instrument first was almost invariably on the contralateral brain side of that predicted to contain the executive system, based upon the subject's MRI calibrated hemisity [13]. This is illustrated in Table 1, where it may be seen that of the 87 of 91 subjects $(96 \%)$ able to report flash asymmetry, $86(99 \%)$ of these reported it to be the side that was correlated with their hemisity $(r=0.97, p=0.000)$. Subjects using the two placebo hemisometers reported only one flash $(n=22)$. The response of thirty seven subjects $(41 \%)$ indicated an observer located in the right hemisphere, while for forty nine $(54 \%)$ it was on the left. Thus, for 86 of the 91 subjects $(96 \%)$, the "observers" were on the same side as the right or left hemisity subtype for that individual. 
Table 1. Agreement of Hemisometer-Determined Observer Side with Hemisity of Subjects

\begin{tabular}{|c|c|c|c|c|}
\hline Subject Hemisity* (n) & $\begin{array}{c}\text { Double Flash } \\
\text { Reported }\end{array}$ & $\begin{array}{c}\text { Single Flash } \\
\text { Reported }\end{array}$ & Side of First Flash (n) & Executive Observer Side (\%) \\
\hline RM (20) & 18 & - & Left (18) & Right $(90 \%)$ \\
Unknown $(10 \%)$
\end{tabular}

Legend: * = MRI calibrated. RM (right brain-oriented male), RF (right brain-oriented female), LM (left brain-oriented male), LF (left brain-oriented female),

Table 2. Correlations of Hemisometer Results with Other Subject Variables:

\begin{tabular}{|c|c|c|c|}
\hline Hemisometer Results vs: & r & p & n \\
\hline Sex & -0.21 & 0.051 & 91 \\
Hemisity from tests-questionnaires & $0.81^{*}$ & 0.000 & 91 \\
Hemisity from larger vgACC side & $0.84^{*}$ & 0.000 & 67 \\
Corpus callosum size & $-0.28^{*}$ & 0.022 & 67 \\
\hline Handedness, claimed & -0.11 & 0.349 & 81 \\
Handedness, any use of left append. & -0.03 & 0.783 & 81 \\
Pen Grasp Type & 0.14 & 0.211 & 81 \\
Emotion Side < & 0.20 & 0.077 & 79 \\
\hline Best Hand Test & $0.75^{*}$ & 0.000 & 88 \\
Phased Mirror Tracing & $0.80^{*}$ & 0.000 & 77 \\
\hline Dichotic Deafness Test & $0.49^{*}$ & 0.000 & 70 \\
\hline Polarity Questionnaire, Score & $0.62^{*}$ & 0.000 & 84 \\
Preference Questionnaire, Score & $-0.31^{*}$ & 0.010 & 67 \\
\hline
\end{tabular}

* = significant ${ }^{\wedge}=$ from Morton and Rafto, 2006, same subjects. $<=$ from Morton, 2003a, same subjects.

As indicated in Table 2, flash hemisometer results were significantly correlated with the larger side of the ventral gyrus of the anterior cingulate cortex $(\mathrm{r}=0.84, \mathrm{p}=0.000, \mathrm{n}$ $=67)$. They were also highly correlated with each of the six secondary methods earlier used for the determination of subject hemisity. That is, for the three biophysical methods, Best Hand Test $(n=88$; [23] Phased Mirror Tracing $(n=77$; [22], and Dichotic Deafness Test ( $\mathrm{n}=70$; [21], coefficients of $0.75,0.80$, and 0.47 were obtained each with $p$ values of 0.000 . Similarly, for three preference questionnaires, Polarity Questionnaire $(\mathrm{n}=84 ;$ [21], Preference Questionnaire $(\mathrm{n}=67 ;[21,31]$, and Asymmetry Questionnaire $(\mathrm{n}=77 ;$; 24$]$, $\mathrm{r}$ values were $0.62,-0.31,0.58$, also with $\mathrm{p}=0.000$, except for the Preference Questionnaire $(\mathrm{p}=0.01)$.

Hemisometer results were not significantly correlated with sex [23, 19], handedness [24, 19], pen grasp [23, 19], emotional side [22], or language laterality. Hemisometry "observer side" outcomes were somewhat correlated with previously determined midline corpus callosal cross sectional areas of these subjects $(r=-0.28, p=0.022)$ [19]. Hemisometer sidedness was very highly correlated with subject hemisity $(\mathrm{r}=0.81, \mathrm{p}=0.000)$, and most highly correlated with $\mathrm{vgACC}$ laterality $(\mathrm{r}=0.84, \mathrm{p}=0.000)$.

\section{Discussion}

This study capitalized upon an earlier discovery of the inability of the nervous system to compensate for time delays due to unequal neural path lengths $[25,26,27]$. That is, when Klemm mildly shocked subjects simultaneously on their head and toe, each invariably reported that they perceived that the shock to their toe to come after the one given to their head. Efron [26] extended this by reporting that laterality differences in perception also appeared for subjects receiving two $1 \mathrm{~ms}$ shocks to their right and left index fingers [27]. He then duplicated major outcomes of his shock studies by using $1 \mathrm{~ms}$ light flashes, separately delivered at various times to the left and right nasal retina of normal subjects.

Here, simultaneous flashes $(0.5 \mathrm{~ms})$ to the right and left hemispheres by either a nasal or temporal retinal flash hemisometer, resulted in 87 of 91 subjects (96\%) reporting two, time-separated, lateral flashes. Of these, $95 \%$ reported the first flash to be on the left (right sided executive) and $94 \%$ reported the first flash appeared on their right (left sided executive). Thus, the flash side asymmetries reproducibly reported by each subject for the two functional hemisometers appeared randomly distributed across the subject population. However, bringing order to this apparent chaos, the side of the brain executive observer indicated by the flash laterality reported was found to be highly correlated of with the larger side of the $\operatorname{vgACC}$ as determined by MRI and with the known hemisity the subjects. This crucial result is consistent with the concept of a unilateral executive system. The highly correlation of 
subject hemisity subtype distributions and observer laterality also reinforced the validity of these hemisometer results and those of earlier methodology $[20,21,22,23$, 24].

Based upon this evidence strongly supporting the existence of an executive element, idiosyncratically placed on either the left or right side of our brains [41], many interesting genetic questions arise, some of which already have been partially answered. Here, the right or left hemisity subtype will be used as an appropriate shorthand term to indicate right or left brain-oriented executive laterality.

In reproductive mating, obviously there are four possible brain laterality pairs: RM-RF (Right brain-oriented Male-Right brain-oriented Female), LM-LF, RM-LF, and LM-RF. It has been observed in 91 mating pairs that for two thirds of them "opposites attract" matings were present That is, $67 \%$ were either RM-LF or LM-RF couples. Same-same hemisity matings were present in the remaining third of the couples (either RM-RF, or LM-LF) $[32,33]$. It has also been found that among these opposite hemisity pairs, RPs (right brain oriented persons) were behaviorally dominant over their supportive LP partners $[32,33]$. Further, offspring from the opposites attract pairs appeared to be "true-breeding", where the hemisity of the children was "like mother, like daughter" and "like father, like son" $[32,33]$. These and other observations have led to the hypothesis that there are two ancient and currently unrecognized human species: the male-dominant Homo sapiens patripolaris, (RM-LF), and the female dominant Homo sapiens matripolaris, (RF-LM) [32,33].

Surviving offspring from the same hemisity RM-RF and LM-LF apparently unnatural pairs were cross-species hybrids and were found to be random in hemisity. Strikingly, many tended to manifest one of the following anomalies: dyslexia, bisexuality, homosexuality, pedophilia, and possibly schizophrenia $[32,33]$. In addition, the dyslexics tested were always RPs, while all bisexuals tested were LPs [32, 34].

The topic of Familial Polarity traces the enlightening history of the two human species from their ancient evolutionary origins to our present society [32, 34]. However, this tale of conflict is beyond the scope of this article.

\section{Conclusions}

The most parsimonious explanation for the congruence of these hemisometer, hemisity, and MRI results was that the observer of the executive system is unilaterally located either on the right or left side of the head. The light flash traversing the shortest neural path to the observer was perceived as arriving first, followed by a second flash whose delay was caused by the longer neural path and multiple additional synaptic steps that must be must traversed from the observer-less side of the brain on across the corpus callosum to reach the observer side. Although the corpus callosal transit times reported have ranged from 2 to $18 \mathrm{~ms}[26,35,30]$ here it probably exceeds $30 \mathrm{~ms}$, the time apparently required to recognize two stimuli as separate $[28,29]$.

The finding that observer side varies with subject appears to resolve the above conflicting reports regarding sidedness of the executive system. That is, the site of the observer of an individual is inherently located either on the left or right side of the brain, independent of the side of their language. Apparently, many functional brain asymmetries are sorted semi independently. There are even reports that language skill and spatial skill are sorted independently, amazingly with both occasionally residing in the same side of the brain [35]. As demonstrated here an executive system laterality also exists, and appears to be sorted independently of either handedness, or language dominance. Thus, these hemisometer, hemisity, and MRI results support not only the concept of executive laterality, but also provide the missing rationale for the existence of hemisphericity [17], and now, for hemisity [13, 33, 36, 37, 38].

\section{Acknowledgements}

The author thanks his many enthusiastic subjects who participated in this unfunded work.

\section{Figure Legend}

Figure 1 Legend: Asymmetries of the Anterior Cingulate Cortex

Three MRI sagittal images were taken for each of the 149 hemisity-calibrated subjects: $6 \mathrm{~mm}$ right, at midline, and $6 \mathrm{~mm}$ left of the midline. (R-bom) right brain-oriented male subject with a larger right $\operatorname{vgACC}$, (R-bof) right brain-oriented female subject with a larger vgACC, (L-bom) left brain-oriented male subject with a larger left vgACC, and (L-bof) left brain oriented female subject with a larger vgACC. Pairs of arrows reaching from the lower surface of the corpus callosum to the cingulate sulcus (CS) illustrate four measurements made for each subject. Corpus callosal thicknesses were also measured for each subject and subtracted from the four measurements to give thickness of the vgACC. The paracingulate sulcus (PCS), when present, is seen above the CS. Note that the distance to the cingulate suclus was shorter on the side of the brain where the paracingulate gyrus was present, while the relative vgACC thickness was greater on the opposite side. 


\section{REFERENCES}

[1] Gazzaniga, M.S., (1995). Principles of human brain organization derived from split-brain studies. Neuron, 14, 217-228.

[2] Lehericy, S., Cohen, L., Baxin, B., Samson, S., Giacomini, E., Rougetet, R., Hertz-Pannier, L., LeBihan, D., Marsault, C., Baulac, M., (2000). Functional MR evaluation of temporal- frontal language dominance compared with the Wada Test. Neurology., 54, 1625-1633.

[3] Riva, D. \& Giorgi, C., (2000). The cerebellum contributes to higher functions during development: evidence from a series of children surgically treated for posterior fossa tumors. Brain, 123, 1051- 1061.

[4] Cheesman, A.L., Barker, R.A., Lewis, S.J., Robbins, T.W., Owen, A.M., Brooks, D.J., (2005). Lateralisation of striatal function: Evidence from 18F-dopa PET in Parkinson's disease. Journal of Neurology. Neurosurgery and Psychiatry, 76, 1204-10.

[5] Libet, B., Gleason, C.A., Wright, E.W., Pearl, D.K., (1983). Time of conscious intention to act in relation to onset of cerebral activity (readiness-potential): The unconscious initiation of a freely voluntary act. Brain, 106, 623-642.

[6] Wegner, D.M., (2002). The illusion of the conscious will. MIT Press, Cambridge, MA

[7] Vogt, B.A., Finch, D.M., Olson, C.R., (1992). Functional heterogeneity in the cingulate cortex: the anterior executive and posterior evaluative regions. Cerebral Cortex 6, 435-443.

[8] Devinsky, O., Morrell, M.J., Vogt, B.A., (1995). Contributions of anterior cingulate cortex to behaviour. Brain, 118, 279-306.

[9] Carter, C.S., Botvinick, M.M., Cohen, J.D., (1999). The contribution of the anterior cingulate cortex to executive processes in cognition. Reviews of Neuroscience, 10, 49-57.

[10] MacDonald, A.W., Cohen, J D., Stenger, V.A., Carter, C.S., (2000). Dissociating the role of the dorsolateral prefrontal and anterior cingulate cortex in cognitive control. Science, 288, 1835- 1838 .

[11] Paus, T., (2001). Primate anterior cingulate cortex: where motor control, drive and cognition interface. Nature Reviews Neuroscience, 2, 417-24.

[12] Fornito A., Wood, S.J., Whittle, S., Fuller, J., Adamson, C., Saling, M.M., Velakoulis, D., Pantelis, C., Yucel, M., (2008). Variability of the paracingulate sulcus and morphometry of the medial frontal cortex: Associations with cortical thickness, surface area, volume, and sulcal depth. Human Brain Mapping, 29, 222-236.

[13] Morton, B.E., Rafto, S.E., (2010). Behavioral laterality advance: Neuroanatomical evidence for the existence of hemisity. Personality and Individual Differences, 49, 34-42.

[14] Herrmann, M., Rotte, M., Grubich, C., Ebert, A.D., Schiltz, K., Munte, T.F., Heinze, H.J., (2001). Control of semantic interference in episodic memory retrieval is associated with an anterior cingulate-prefrontal activation pattern. Human Brain Mapping, 13, 94-103.
[15] Stephan, K.E., Marshall, J.C., Friston, K.J., Rowe, J.V., Ritzl, A., Zilles, K., Fink, G. R., (2003). Lateralized cognitive processes and lateralized task control in the human brain. Science, 301, 384-386.

[16] Sperry, R.W., (1968). Hemisphere disconnection and unity in conscious awareness. American Psychologist, 23, 723-733.

[17] Beaumont, G., Young, A., McManus, I.C., (1984). Hemisphericity: A critical review. Cognitive Neuropsychology 1, 191-212.

[18] Morton, B.E., (2012a). Right and left brain oriented individuals show opposite behavioral preferences. Frontiers of Physiology, 3, 407-419

[19] Morton, B.E., Rafto, S.E., (2006). Corpus callosum size is linked to dichotic deafness and hemisphericity, not sex or handedness. Brain and Cognition, 62, 1-8.

[20] Morton, B.E., (2001). Large individual differences in minor ear output during dichotic listening. Brain and Cognition, 45, 229-237.

[21] Morton, B.E., (2002). Outcomes of hemisphericity questionnaires correlate with unilateral dichotic deafness. Brain and Cognition, 49, 63-72.

[22] Morton, B.E., (2003a). Phased mirror tracing outcomes correlate with several hemisphericity measures. Brain and Cognition, 51, 294-304.

[23] Morton, B.E., (2003b). Two-hand line-bisection task outcomes correlate with several measures of hemisphericity. Brain and Cognition, 51, 305-316.

[24] Morton, B.E., (2003c). Asymmetry Questionnaire outcomes correlate with several hemisphericity measures. Brain and Cognition, 51, 372-374

[25] Klemm, O., (1925). Ueber die wirksamkeit kleinster zeitunterschiede [On the effect of the smallest time differences]. Archives Gesamter Psychology, 50, 204-209.

[26] Efron, R., (1963a). The effect of handedness on the perception of simultaneity and temporal order. Brain, 86 , 261-284

[27] Efron, R., (1963b). The effect of stimulus intensity on the perception of simultaneity in right- and left-handed subjects. Brain, 86, 285-294.

[28] Corballis, M.C., (1996). Hemispheric interactions in temporal judgments about spatially separated stimuli. Neuropsychology, 10, 42-50.

[29] Stanford, T.R., Shankar, S, Massoglia, D.P., Costello, M.G., Salinas, E., (2010). Perceptual decision making in less than 30 milliseconds. Nature Neuroscience, 13, 379-386.

[30] Terasaki, O., Okazaki, M., (2002). Transcallosal conduction time measured by hemifield stimulation of face images. Neuroreport 13, 97-99

[31] Zenhausern, R., (1978). Imagery, cerebral dominance, and style of thinking: A unified field model. Bulletin of the Psychonomic Society, 12, 381-384.

[32] Morton, B.E., (2011). Neuroreality. Megalith Books, Doral, FL. 
[33] Morton, B.E., (2012b). Two human species exist. Megalith Books, Doral, FL.

[34] Morton, B.E., (2013). Behavoral laterality of the brain: support for the binary construct of hemisity. Frontiers of Psychology, 4 (doi: 10.3387/fpsyg.2013.00683), 1-12

[35] Schnitzler, A., Kessler, K.R. \& Benecke, R., (1996). Transcallosally mediated inhibition of interneurons within human primary motor cortex. Experimental Brain Research, 112, 381-91.

[36] Floel, A., Jansen, A., Deppe, M., Kanowski, M., Konrad, C., Sommer, J., Knecht, S., (2005). Atypical hemispheric dominance for attention: functional MRI topography. Journal of Cerebral Blood Flow and Metabolism 25, 1197-1208.
[37] Morton, B.E., (2014). Further Evidence for Hemisity Sorting during Career Specialization, Journal of Career Assessment, 22, 317-328.

[38] Morton, B.E. (2017). The dual quadbrain and modular consciousness. Universal Journal of Psychology, 5, 157-166.

[39] Morton, B.E., (2003d). Hemisphericity of university students and professionals: Evidence for sorting during higher education. Brain and Cognition, 52, 319-325.

[40] Dolan, R. J., (2002). Emotion, cognition, and behavior. Science, 298, 1191, 1194.

[41] Bogen, J.E., (1969). The other side of the brain, IV, The $A / P$ ratio. Bulletin of the Los Angeles Neurological Society, 34, 135-162. 